\title{
Epicardial off-pump pulmonary vein isolation and vagal denervation improve long-term outcome and quality of life in patients with atrial fibrillation
}

\author{
Louise Bagge, MD, ${ }^{\text {a }}$ Per Blomström, MD, PhD, ${ }^{\text {a }}$ Leif Nilsson, MD, PhD, ${ }^{\mathrm{b}}$ Gunnar Myrdal Einarsson, MD, PhD,
}

Lena Jidéus, $\mathrm{MD}, \mathrm{PhD},{ }^{\mathrm{b}}$ and Carina Blomström-Lundqvist, $\mathrm{MD}, \mathrm{PhD}^{\mathrm{a}}$

Objectives: The limited information available on thoracoscopic pulmonary vein isolation combined with ganglionated plexi ablation and the lack of studies regarding its effect on quality of life and physical capacity urged us to study its acute and long-term results in patients with atrial fibrillation.

Methods: Forty-three patients (mean age 57.1 years) with symptomatic atrial fibrillation referred for thoracoscopic off-pump epicardial pulmonary vein isolation and ganglionated plexi ablation using radiofrequency energy were included.

Results: The physical capacity improved significantly at 6-month follow-up compared with baseline (mean \pm standard deviation, 165.2 \pm 65 Watt versus $155.9 \pm 57$ Watt, $P=.02$ ). Quality of life (Short Form-36 health survey) significantly improved 12 months after surgery compared with baseline in all subscales except for bodily pain. The symptom severity questionnaire score decreased significantly from mean $15.2 \pm 4.0$ points to $10.7 \pm$ 4.8 points $(P=.02)$. Overall, 25 of 33 patients $(76 \%)$ followed up for 12 months had no symptomatic atrial fibrillation recurrences or atrial fibrillation episodes on 24-hour Holter recordings. The corresponding figures were $79 \%(19 / 24)$ for patients with paroxysmal atrial fibrillation, $100 \%$ (2/2) for persistent atrial fibrillation, and 57\% (4/7) for permanent atrial fibrillation. The most common complication was bleeding events $(9 \%)$ during pulmonary vein dissection.

Conclusions: Epicardial off-pump pulmonary vein isolation combined with ganglionated plexi ablation improved quality of life, symptoms, and exercise capacity and therefore may be considered for patients with atrial fibrillation who fail endocardial pulmonary vein ablation or as a first-line procedure if left atrial appendage exclusion is warranted.

Although transvenous radiofrequency $(\mathrm{RF})$ atrial fibrillation (AF) ablation is reported to eliminate AF in a high proportion of cases, ${ }^{1,2}$ pulmonary vein $(\mathrm{PV})$ reconduction is still a recognized problem, possibly reflecting difficulties in achieving transmural lesions. ${ }^{3}$ Alternative approaches, such as thoracoscopic epicardial PV isolation, therefore have evolved. This technique was recently reported to result in AF elimination in $80 \%$ to $91 \%$ of the patients. ${ }^{4,5}$ Furthermore, it was recently suggested that ganglionated plexi (GP) ablation combined with epicardial PV isolation and left atrial appendage (LAA) exclusion may increase the rate of freedom from AF. ${ }^{6-10}$ Limited information is, however, available about the long-term outcome of such procedure, and its effects on quality of life (QoL), symptoms, and physical capacity are lacking. The purpose of the present study therefore was to describe the acute and long-term results of combined PV isolation and GP ablation for the treatment of AF

From the Departments of Cardiology and Thoracic Surgery, ${ }^{\mathrm{b}}$ Uppsala University Hospital, Uppsala, Sweden.

Received for publication Aug 14, 2008; revisions received Nov 19, 2008; accepted for publication Dec 19, 2008.

Address for reprints: Louise Bagge, MD, Department of Cardiology, Uppsala University Hospital, 75185 Uppsala, Sweden (E-mail: louise.bagge@medsci.uu.se).

J Thorac Cardiovasc Surg 2009;137:1265-71

$0022-5223 / \$ 36.00$

Copyright $\subset 2009$ by The American Association for Thoracic Surgery

doi:10.1016/j.jtcvs.2008.12.017 with regard to QoL, symptoms, physical capacity, safety, and freedom from AF.

\section{METHODS \\ Patients and Investigations}

A total of 43 patients were referred for epicardial $\mathrm{PV}$ isolation combined with GP ablation between November 2005 and March 2008 (Table 1). Patients who had failed at least 1 class I (86\%) or III $(93 \%)$ antiarrhythmic agent for symptomatic paroxysmal, persistent, or permanent AF were included. Eighteen $(42 \%)$ patients had previously failed a transvenous endocardial PV isolation, and 11 patients $(26 \%)$ previously had an atrial flutter ablation. Six patients (14\%) had a permanent pacemaker preoperatively.

All patients were treated with warfarin with the international normalized ratio level targeted between 2 and 3 for at least 1 month prior to the procedure. Thrombus formation in the LAA was excluded by transesophageal echocardiography the day before the operation. Warfarin was replaced by low-molecular-weight heparin when the international normalized ratio value was less than 2 before surgery but was resumed on the evening before operation. It was then withdrawn 3 months after the operation except for patients with a history of stroke or transient ischemic attack or with CHADS2 score above 2. Heparin (5000 IU) was administrated intravenously during surgery. Antiarrhythmic drugs (AADs) were continued for at least 3 months after surgery, at which time they were withdrawn in patients who remained in sinus rhythm. Amiodarone was prescribed if AF recurred more than once during the first 3 months postoperatively.

The clinical evaluation at baseline included a symptom severity questionnaire (SSQ), Study Short Form-36 questionnaire for QoL, ${ }^{11}$ physical examination, 12-lead electrocardiogram (ECG), 2-dimensional echocardiography, exercise bicycle test, coronary angiography (if age above 50 years 


$$
\begin{aligned}
& \text { Abbreviations and Acronyms } \\
& \text { AAD }=\text { antiarrhythmic drug } \\
& \text { AF }=\text { atrial fibrillation } \\
& \text { CT }=\text { contrast tomography } \\
& \text { ECG }=\text { electrocardiogram } \\
& \text { GP }=\text { ganglionated plexi } \\
& \text { LAA }=\text { left atrial appendage } \\
& \text { PV }=\text { pulmonary vein } \\
& \text { QoL }=\text { quality of life } \\
& \text { RF }=\text { radiofrequency } \\
& \text { SSQ }=\text { symptom severity questionnaire }
\end{aligned}
$$

or if symptoms suggested ischemic heart disease), 24-hour Holter recording, and contrast tomography (CT) for PV anatomy. Coronary artery disease was defined as previous myocardial infarction, coronary artery revascularization, or documented significant coronary artery stenosis on angiography. The 5 variables in the SSQ (palpitations, fatigue, dizziness, lack of energy, dyspnea) were each scored with regard to severity on a 5 -grade scale. ${ }^{2}$ The patients were followed with regard to symptoms of AF and 12-lead ECG at 3,6 , and 12 months after the surgery. The exercise bicycle test, echocardiography, CT (to exclude PV stenosis), 24-hour Holter recording, SSQ, and Short Form-36 were evaluated after 6 months, and the latter three (24-hour Holter recording, SSQ, and Short Form-36) were repeated at 12-month follow-up. A successful outcome was defined as no documented symptomatic AF episodes or left atrial tachycardias after 12 months of follow-up, excluding the initial 3 months postoperatively, according to 12-lead ECG, 24-hour Holter recording, and spot ECG recorded when symptoms dictated. Patients gave their consent to the study, which was approved by the local Ethics Review Board.

\section{Procedural Technique}

The thoracoscopic off-pump procedure included epicardial PV isolation, left atrial GP ablation, division of the ligament of Marshall, and left atrial appendectomy. General anesthesia was administered with a double-lumen endotracheal tube. The procedure started on the right side in all but 1 patient to ensure that electrical isolation could be completed for all veins, in case the excision of the LAA would result in any complication.

With the patient in a left lateral recumbent position and with the right lung deflated, the procedure was initiated by inserting the thoracoscope (Storz, Tuttlingen, Germany) at the level of the xiphoid process. A small thoracic incision was made in the third intercostal space to approach the right PVs. Through the incision, the pericardium was opened anterior and parallel to the phrenicus nerve from the superior vena cava down to the inferior vena cava. Blunt dissection around the PVs was performed with a lighted dissector (first-generation device, Atricure, West Chester, Ohio) entering into the oblique sinus and passing out above the superior PV. The dissector was then replaced by a rubber band.

To identify predominantly vagal GP, high-frequency electrical stimulation ( 800 pulses per minute, pulse-width $9.9 \mathrm{~ms}$ ) was applied at 10 sites according to a map (Figure 1, $A$ ) adapted from Warren Jackman, University of Oklahoma. A positive response was defined as a bradycardia with a doubling of the R-R interval. The rubber band was then used to introduce the ablation clamp (Atricure) around the PV pair. PV conduction prior to ablation was assessed for each PV pair. The clamp was closed well proximal to the confluence of the veins where after RF ablation was applied according to an algorithm that automatically stopped the ablation when the impedance change indicated that a transmural lesion was achieved between the jaws. Conduction block was then confirmed by pacing or, if AF was present, by the absence of fibrillation potentials on the distal or PV side of the ablation line. In case conduction block was not achieved after the first 3 consecutive ap-
TABLE 1. Patient demographics

\begin{tabular}{lc}
\hline \multicolumn{1}{c}{ Characteristics } & Results \\
\hline Age (y), median (range) & $58(36-71)$ \\
Sex, male & $29(67)$ \\
History of AF (y), median (range) & $7(1-22)$ \\
Paroxysmal AF & $28(65)$ \\
Persistent AF & $6(14)$ \\
Permanent AF & $9(21)$ \\
Lone AF & $21(49)$ \\
Hypertension & $13(30)$ \\
Cerebral stroke/TIA/neurologic deficit & $8(19)$ \\
Diabetes mellitus & $1(2)$ \\
Coronary artery disease & $3(7)$ \\
Valvular heart disease & $4(9)$ \\
Heart failure or cardiomyopathy & $6(14)$ \\
Asthma & $4(9)$ \\
Sleep apnea syndrome & $2(5)$ \\
LVEF $<$ 50 & $6(14)$ \\
LAd, cm, median (range) & $4.5(2.6-6.2)$ \\
\hline
\end{tabular}

Figures denote numbers and percentages in parentheses unless otherwise stated. $A F$, atrial fibrillation; TIA, transient ischemic attack; $L V E F$, left ventricular ejection fraction; $L A d$, left atrial diameter.

plications, 1 or 2 additional RF applications were given until PV conduction block occurred. The high-frequency stimulation scheme (Figure 1, $A$ ) was then repeated, and if GP activity was still present, it was abolished by electrocautery or by an RF ablation pen (Atricure), until no vagal response could be provoked. A thoracic drain was inserted, the lung was inflated, and the incisions closed.

The patient was then repositioned in a right lateral recumbent position and the procedure repeated as described above to approach the left PVs for isolation and GP ablation. If a ligament of Marshall could be identified, it was divided. Finally, the LAA was excised using a stapler (Autosuture, United States Surgical, Norwalk, Conn), if judged feasible and safe (absence of a wide base or thin wall). Intercostal analgesic blockade was given on both the right and left sides.

\section{Statistical Analysis}

Continuous variables, when appropriate, were compared with the use of Student $t$ test or otherwise Mann-Whitney $U$ test. Tukey HSD test was used for the comparison of QoL. Wilcoxon matched-paired test or, when appropriate, the analysis of variance test was used for the evaluation of symptom scores. All $P$ values were 2 -sided. Results are expressed as mean \pm 1 standard deviation, unless otherwise stated.

The authors had full access to the data and take responsibility for its integrity. All authors have read and agree to the manuscript as written.

\section{RESULTS}

The results are based on the 42 patients in whom PV isolation could be performed. One patient was excluded because of premature termination of the procedure before PV isolation due to bleeding from the right PV during dissection.

Isolation of all PVs was completed in all but 5 of the remaining 42 patients and required 3 to 4 applications (median 3 ) on the right side and 2 to 5 applications (median 3 ) on the left side. Electrical isolation was not achieved on the left side in 1 patient despite 7 applications and not on the right side in another patient despite 11 applications. In 2 cases, PV 


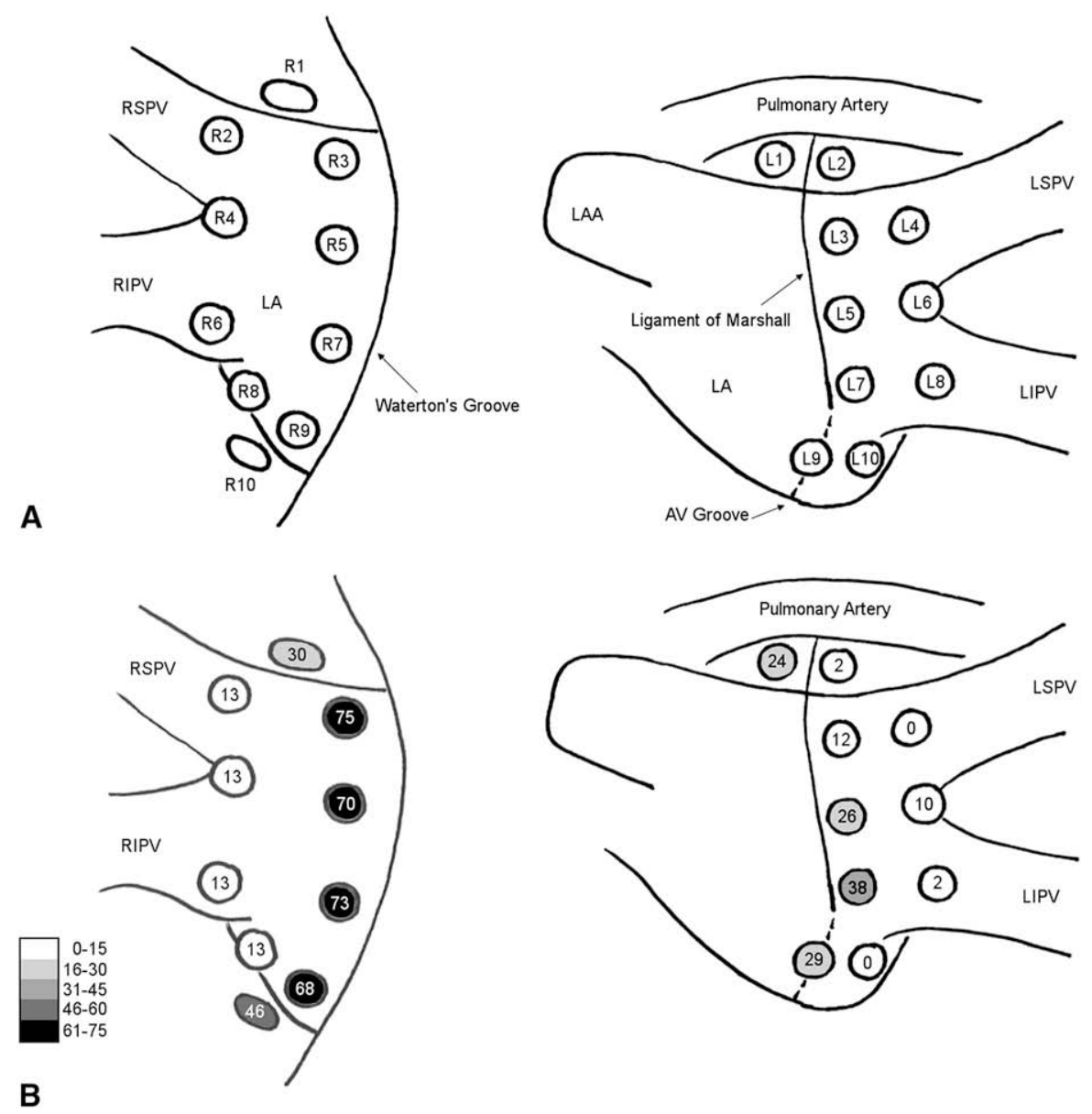

FIGURE 1. A, Sites of high-frequency stimulation of ganglionated plexi (GP). In every patient 10 sites on the right side (R1-R10) and 10 on the left side (L1-L10) of the left atrium were mapped. $R S P V$, Right superior pulmonary vein; $R I P V$, right inferior pulmonary vein; $L S P V$, left superior pulmonary vein; $L I P V$, left inferior pulmonary vein; $L A$, left atrium; $L A A$, left atrium appendage; $A V$, atrioventricular; $R$, right; $L$, left. B, Results of epicardial GP mapping. High-frequency stimulation map showing distribution of active GP sites displayed as percentages as well as by grayscale.

isolation could not be completed on the right side because of either a rift of 1 of the PVs or a rift of the tissue around the right inferior PV and the inferior vena cava during dissection. In the last case, a thrombus in the LAA disclosed by the transesophageal echocardiography during surgery precluded PV isolation on the left side. PV reconduction was confirmed for all vein pairs tested in the patients with a prior catheter ablation procedure.

Vagal GP activity was present in all but 2 patients at baseline before the PV isolation. Active GP at baseline were identified on both the left and right side in 23 of $42(55 \%)$ patients but on the right side only in 17 of $42(40 \%)$ patients. There were significantly more active GP sites on the right than on the left side $(4.0 \pm 2.4$ vs $1.4 \pm 1.7, P<.001)$, and the greatest concentrations of vagal GP activity were found around the Waterton's groove on the right side and the ligament of Marshall on the left side (Figure $1, B$ ). In 1 patient in whom the operation started on the left side, GP activity could only be demonstrated on the right side at baseline.
Patients who previously had transvenous catheter-based AF ablation had significantly fewer active vagal GP sites (mean $4.0 \pm 2.2$ ) at baseline than those who had not (mean $6.4 \pm 3.5, P=.01$ ). Vagal GP activity was present in all but 1 of the patients who had previous AF ablation. The number of active vagal GP sites in patients with paroxysmal AF was not significantly different from those in patients with persistent or permanent $\mathrm{AF}$ (mean $4.9 \pm 3.1$ vs $6.2 \pm 2.0, P=.23$ ). Complete elimination of all active GP sites failed in 16 of the 42 patients $(38 \%)$, and the maximum number of active GP sites that could not be abolished was 4 sites in 1 patient. The ligament of Marshall was identified and divided in 40 patients $(95 \%)$, and the LAA was excluded in 32 of the 42 patients $(76 \%)$. The LAA could not be extirpated because of a wide base in 6 patients and because of poor accessibility in another 3 patients. A thrombus precluded LAA exclusion in another patient.

The surgical procedure time ranged from 185 to 410 minutes (median 227.5 minutes). The hospital stay ranged 

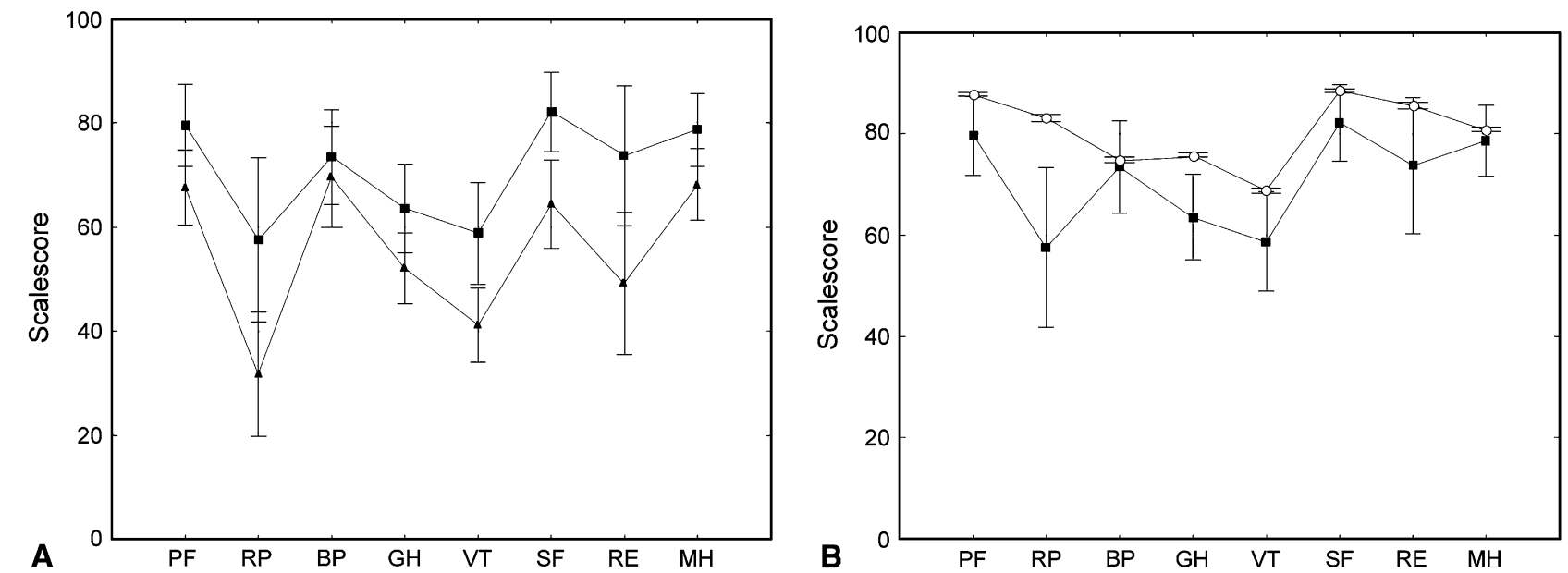

FIGURE 2. Comparison of quality of life assessed by the Short Form-36 questionnaire for QoL. A, Baseline ( $\mathrm{n}=42)$ versus 12 months following surgery $(\mathrm{n}=33)$. Error bars show $95 \%$ confidence limits of the mean. $\boldsymbol{\Lambda}$, Baseline; $\mathbf{\square}, 12$-month follow-up; $P F$, physical functioning; $R P$, role limitation due to physical problems; $B P$, bodily pain; $G H$, general health; $V T$, vitality; $S F$, social functioning; $R E$, role limitation due to emotional problems; $M H$, mental health. PF: $P=.0019$; RP: $P=.0078$; BP: $P=.39$; GH: $P=.0070$; VT: $P=.00014$; SF: $P=.00014$; RE: $P=.00059$; MH: $P=.00036$. B, Twelve months after surgery $(\mathrm{n}=33)$ versus the general Swedish population. $\mathbf{\square}, 12$-month follow-up; $\bigcirc$, Swedish general population. PF: $P=.038$; RP: $P=.0023$; BP: $P=.78 ; \mathrm{GH}: P=.0065 ; \mathrm{VT}: P=.046 ; \mathrm{SF}: P=.096 ; \mathrm{RE}: P=.080 ; \mathrm{MH}: P=0.53$.

from 6 to 34 days (median 11 days) including 2 to 17 days (median 4 days) at the intensive care unit.

\section{Follow-up}

Forty-one of the 42 patients who had PV isolation were followed after surgery. One patient living abroad was excluded from follow-up.

Twenty-five of the 33 patients $(76 \%)$ who had completed the 12-month follow-up schedule were free from $\mathrm{AF}$ episodes according to 12-lead ECG, 24- to 48-hour Holterrecording, and spot ECG at the time of symptoms. When success rate was related to the type of $\mathrm{AF}$, the corresponding figures were $79 \%$ (19 of 24) for patients with paroxysmal $\mathrm{AF}, 100 \%$ (2 of 2) for those with persistent $\mathrm{AF}$, and $57 \%$ (4 of 7) for patients with permanent AF, although the differences did not reach statistical significance. The rate of freedom from AF was $69 \%$ in patients who had a previous catheter-based $\mathrm{AF}$ ablation compared with $81 \%$ in those who had not $(P=.46)$. None of the 25 patients who were free from clinical recurrent $\mathrm{AF}$ episodes had asymptomatic episodes of AF on Holter recordings, but in 5 of them the recording showed supraventricular extrasystoles or nonsustained regular supraventricular tachycardia. No patient suffered from left atrial tachycardias.

Among the 25 patients clinically free from $\mathrm{AF}$ at 12-month follow-up, $8(32 \%)$ were still on AADs and 6 were still on warfarin related to earlier stroke/transient ischemic attack/thromboembolic event in 4 patients and for unknown reason in 2 patients.

Of the remaining 9 patients followed up for 12 months, 1 patient missed the 24-hour Holter recording related to cancer treatment but had no clinical or symptomatic AF recurrences, 6 patients had paroxysmal AF, and 2 had permanent AF. Two other patients had tricuspid isthmus ablation for atrial flutter. Another 2 patients required pacemaker implantation for chronotropic incompetence and bradycardia related to medication with beta-blocking agents 18 months and 8 months after surgery, respectively.

Of the remaining 7 patients who had not reached 12 months of follow-up, 3 of 4 patients followed up for 6 months and 2 out of 3 patients who completed 3 months of follow-up had no documented episodes of AF.

The number of active GP sites at baseline did not differ between patients who remained free from $\mathrm{AF}(5.1 \pm 3.3)$ and those with AF recurrence at last follow-up (5.2 \pm 1.9 , $P=.864$ ).

Health-related QoL was significantly improved at 6 and 12 months on all scales except for bodily pain, as compared with baseline before surgery (Figure 2, A). Physical functioning and role limitation due to physical problems was not significantly improved until 12-month follow-up. At 12-month follow-up, QoL reached the values of a general Swedish population for 4 of the scales (Figure 2,B). The QoL prior to surgery was significantly lower than for the Swedish general population, with the exception of bodily pain.

Overall, symptom severity scores improved significantly at 12 months after surgery (10.7 \pm 4.8 points) compared with baseline (15.2 \pm 4.0 points, $P=.02)$. All symptoms except for dizziness and palpitations improved at 12-month follow-up as compared with baseline (Table 2). Patients who remained in sinus rhythm had significantly lower symptom severity score at 12-month follow-up (mean $10.2 \pm 5.0$ 
TABLE 2. Symptoms at baseline and at 6 and 12 months after the surgery

\begin{tabular}{|c|c|c|c|c|c|}
\hline \multirow[b]{2}{*}{ SSQ variable } & \multirow[b]{2}{*}{ Baseline $(n=37)$, mean $(95 \%$ CL) } & \multicolumn{2}{|c|}{6 mo $(n=17)$} & \multicolumn{2}{|c|}{$12 \mathrm{mo}(\mathrm{n}=33)$} \\
\hline & & Mean $(95 \%$ CL) & $P$ value* & Mean $(95 \%$ CL $)$ & $\boldsymbol{P}$ value $\dagger$ \\
\hline Palpitations & $2.6(2.1-3.1)$ & $1.8(1.1-2.4)$ & .09 & $2.2(1.7-2.6)$ & .28 \\
\hline Fatigue & $3.6(3.2-4.1)$ & $2.2(1.5-3.0)$ & .018 & $2.2(1.7-2.7)$ & .004 \\
\hline Dizziness & $2.1(1.7-2.5)$ & $1.9(1.2-2.7)$ & .35 & $1.8(1.4-2.3)$ & .67 \\
\hline Lack of energy & $3.9(3.5-4.3)$ & $2.5(1.6-3.4)$ & .07 & $2.5(2.0-3.0)$ & .006 \\
\hline Dyspnea & $2.9(2.5-3.4)$ & $1.9(1.2-2.7)$ & .07 & $2.0(1.5-2.4)$ & $<.001$ \\
\hline
\end{tabular}

$C L$, Confidence limit; SSQ, symptom severity questionnaire. *SSQ score at 6 mo vs baseline. $\dagger$ SSQ score at 12 mo vs baseline.

points) compared with baseline (mean $15.8 \pm 4.7$ points, $P=.036)$, but only dyspnea $(P=.018)$ and fatigue $(P=$ $.015)$ improved significantly. In patients with recurrences of $\mathrm{AF}$, the symptom severity score did not differ between 12-month follow-up and baseline (mean $13.4 \pm 3.2$ points vs $15.0 \pm 1.6$ points, $P=.42$ ), and nor did the separate symptoms.

The physical capacity on exercise test was significantly improved at 6 months compared with baseline (165.2 \pm 64.8 Watt vs $155.9 \pm 56.7$ Watt, $P=.02$ ).

\section{Learning Curve}

The first 40 procedures were divided into groups of 10 to analyze the learning curve with regard to procedure time and bleeding events (Figure 3 ). The procedure time was significantly reduced from $274 \pm 42$ minutes for the first 10 procedures to $224 \pm 26$ minutes for the last 10 procedures $(P=.0070)$.

\section{Complications}

There were no perioperative or late deaths after surgery. Major bleeding, defined as bleeding requiring transfusion or surgical intervention and/or leading to treatment cessation, occurred in 6 patients (Table 3). The reasons for bleeding

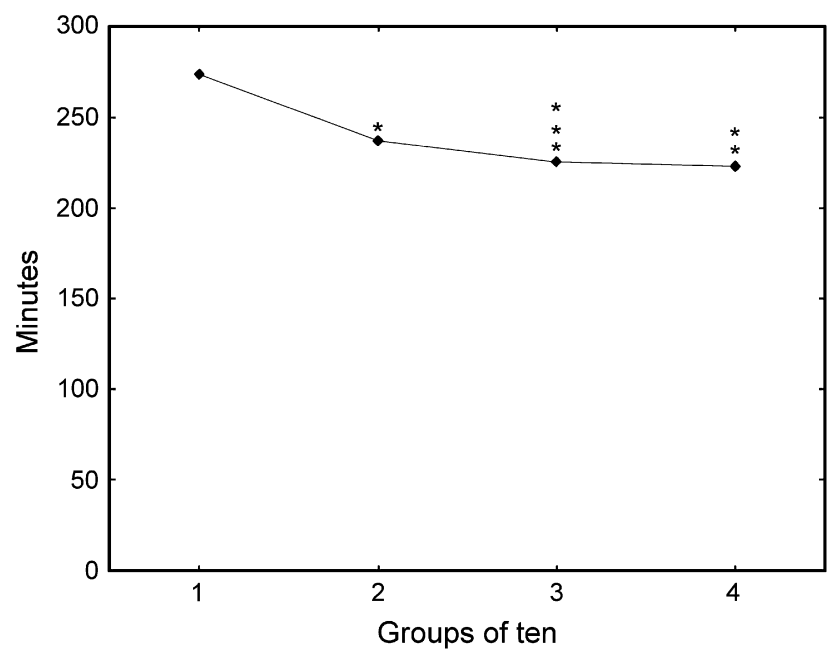

FIGURE 3. Learning curve with procedure time averaged per group of 10 consecutive patients. *The time at which major bleeding occurred.

were rupture of the staple line when the LAA was extirpated (1), rift of a right PV (2), rift of the tissue around the right inferior PV and the inferior vena cava (1), rift of the pulmonary artery during dissection (1), and bleeding from an intercostal artery (1), which required reoperation twice. In 4 of these cases, an extension of the incision was made, and in 2 , the heart-lung machine was required. In the first patient requiring heart-lung machine, the femoral nerve was damaged during the acute dissection for cannulation in the groin, and in the other, the operation could not be completed. Three of these patients needed blood transfusions. The time at which bleeding events occurred is depicted in the learning curve (Figure 3). One patient with permanent AF suffered from a stroke 2 days after the operation. Warfarin had been discontinued 4 days before the surgery but by mistake was not replaced by low-molecular-weight heparin until the day before operation. No patient developed PV stenosis according to $\mathrm{CT}$ performed 5 to 12 months after surgery in 31 patients.

\section{DISCUSSION}

The new minimally invasive epicardial procedure that combines PV isolation and GP ablation has revealed some impressive success rates in eliminating $\mathrm{AF},{ }^{6-10}$ ranging between $74.2 \%$ and $93 \%$ at 6-month follow-up ${ }^{6,10}$ and $75 \%$ to $90 \%$ at mean 16.6 to 17 months follow-up. ${ }^{7,9}$

TABLE 3. Complications after surgery

\begin{tabular}{ll}
\hline \multicolumn{1}{c}{ Complication } & $\mathbf{n}(\%)$ \\
\hline Perioperative* $(\mathrm{n}=43)$ & \\
Hemorrhage & $6(14)$ \\
Pleural effusion/hemothorax & $2(5)$ \\
Ventricular tachycardia & $1(2)$ \\
Transient confusion & $1(2)$ \\
Rib fracture & $1(2)$ \\
Infections & $4(9)$ \\
Ischemic stroke & $1(2)$ \\
Postpericardiotomy syndrome & $1(2)$ \\
Late $\dagger(n=42)$ & $1(2)$ \\
Postpericardiotomy syndrome & $2(5)$ \\
Infections & $1(2)$ \\
Esophagitis and gastritis & $1(2)$ \\
Incisional hernia &
\end{tabular}


Divergent patient selections, variation in lesion sets used, and different study designs and follow-ups, however, make it difficult to compare the present study with others. In the present study, $75.8 \%$ of the patients were clinically free from AF, which is a lower success rate than the $93 \%$ reported by Mehall et al. ${ }^{6}$ The higher success rate in that study may be explained by their selection of patients with lone AF only and their shorter follow-up of 6 months, which notably was only available for 15 of their 26 patients. The other study reporting a higher success rate $(90 \%)$ only included 20 patients, of whom only $10 \%$ had previous catheter ablation compared with our $42 \%$, and of whom $80 \%$ had paroxysmal AF compared with $65 \%$ in our study. ${ }^{9}$ It is well known that PV isolation is more effective in patients with paroxysmal $\mathrm{AF}$ than in those with persistent $\mathrm{AF},{ }^{1}$ and patients with previously failed transvenous $\mathrm{AF}$ ablation are likely more difficult to treat, as was indicated in our study. Our results are more in accordance with the two other studies, ${ }^{7,10}$ although one study ${ }^{7}$ included only 20 patients and the other ${ }^{10}$ was limited by their short follow-up and in that AADs had not been discontinued in over $42 \%$ of their 66 patients. Furthermore, their autotriggered event monitor, including only 15 -second rhythm strip for calculation of AF burden, is limited by the lack of validity studies. Hence, considering number and selection of patients and time of follow-up, the outcome of the present study compares favorably with previous studies, even though asymptomatic $\mathrm{AF}$ episodes could not be excluded because we did not have access to continuous rhythm monitoring.

Our finding that vagal GP activity is far more prevalent on the right than on the left side as well as around the ligament of Marshall and Waterston's groove has been reported by others. ${ }^{6,7,10}$ One cannot exclude, however, that the preceding right-sided PV isolation may have eliminated some of the active GP sites present on the left side. The importance of GP ablation for an improved outcome of the procedure, however, has not yet been proven. One retrospective study reported that vagal denervation enhances the long-term benefit after circumferential ablation for paroxysmal $\mathrm{AF},{ }^{12}$ and another prospective but nonrandomized study suggested that the addition of GP ablation to conventional PV antrum isolation may increase the success rate from $70 \%$ to $91 \%{ }^{13}$ Others claiming the opposite reported that freedom from AF recurrences was more common after circumferential PV ablation $(63 \%)$ than after anatomic GP ablation as a stand-alone treatment $(26 \%) .{ }^{14}$ Moreover, epicardial fat pad ablation, comparable with GP ablation, did not suppress $\mathrm{AF}$ induction in an experimental study. ${ }^{15}$

Our novel findings that both symptoms and QoL improved after the procedure are of great importance as the main clinical indication for the procedure is to reduce symptoms and increase QoL. The symptom severity scores recorded at baseline in our study were comparable with those reported in a previous randomized trial of transvenous circumferential PV ablation for highly symptomatic longstanding persistent $\mathrm{AF}^{2}$ indicating that our patients were as highly symptomatic. The possibility of a placebo effect after the surgical procedure explaining the differences in symptoms and QoL between baseline and 12 months is contradicted by our observation that the symptom severity score only improved in the patients who remained in sinus rhythm and not in the patients with AF recurrences. The finding that palpitations were not significantly reduced even in patients with sinus rhythm may be related to the presence of extra beats, revealed on Holter recordings. The improved physical capacity following the procedure in our study further indicates that there was not only a subjective but also an objective improvement after surgery.

Major bleeding was the most common complication in our study, which is in accordance with the 4 other studies. Our bleeding rate of $14 \%$ was higher than that reported in 2 other studies $(2.7 \%-4.8 \%)^{7,10}$ but comparable to the $15.0 \%$ in another study. ${ }^{9}$ According to our experience, the most vulnerable moment is the dissection around the PVs, accounting for 4 of the 6 bleeding events, which did not seem to follow the learning curve (Figure 3). Although only 1 bleeding event was related to the exclusion of the LAA, a significant portion of patients $(23.8 \%)$ were judged to be at too high risk for such procedure, indicating that this may also be a critical moment. This is supported by the report of 1 death due to tearing of the base of the LAA. ${ }^{10}$ An advantage with the epicardial approach is the possibility of LAA exclusion, for an anticipated reduced risk of future thromboembolic events, which is not yet feasible with the transvenous AF ablation technique. ${ }^{16}$ The potentially lifethreatening complications associated with transvenous $\mathrm{AF}$ ablation, such as PV stenosis, tamponade, and left atrialesophageal fistula, ${ }^{16,17}$ seem to be of no major concern using the epicardial approach, as to our best knowledge, none of these complications have been reported. However, 1 case of pulmonary embolism requiring rehospitalization has been reported after the procedure. ${ }^{18}$ This less invasive procedure may avoid the use of cardiopulmonary bypass and most likely carries a lower risk for pacemaker implantation. These are possible advantages of this procedure over a traditional cut-and-sew maze procedure.

In conclusion, off-pump epicardial PV isolation combined with GP ablation is a relatively safe and efficacious treatment and may therefore be considered for patients with drug-resistant paroxysmal or persistent $\mathrm{AF}$ who failed endocardial PV ablation or as a first-line procedure if LAA exclusion is warranted. Further studies are, however, necessary to reveal the additive value of GP ablation.

\section{References}

1. Stabile G, Bertaglia E, Senatore G, De Simone A, Zoppo F, Donnici G, et al. Catheter ablation treatment in patients with drug-refractory atrial fibrillation: a prospective, multi-centre, randomized, controlled study (Catheter Ablation For The Cure Of Atrial Fibrillation Study). Eur Heart J. 2006;27:216-21. 
2. Oral H, Pappone C, Chugh A, Good E, Bogun F, Pelosi F Jr, et al. Circumferential pulmonary-vein ablation for chronic atrial fibrillation. $N$ Engl J Med. 2006;354: 934-41.

3. Chen ML, Yang B, Xu DJ, Zou JG, Shan QJ, Chen C, et al. [Electrophysiological findings and ablation strategies in patients with atrial tachyarrhythmias after left atrial circumferential ablation in the treatment of atrial fibrillation]. Zhonghua Xin Xue Guan Bing Za Zhi. 2007;35:119-22.

4. Wolf RK, Schneeberger EW, Osterday R, Miller D, Merrill W, Flege JB Jr, et al. Video-assisted bilateral pulmonary vein isolation and left atrial appendage exclusion for atrial fibrillation. J Thorac Cardiovasc Surg. 2005; 130:797-802.

5. Sagbas E, Akpinar B, Sanisoglu I, Caynak B, Tamtekin B, Oral K, et al. Videoassisted bilateral epicardial pulmonary vein isolation for the treatment of lone atrial fibrillation. Ann Thorac Surg. 2007;83:1724-30.

6. Mehall JR, Kohut RM Jr, Schneeberger EW, Taketani T, Merrill WH, Wolf RK. Intraoperative epicardial electrophysiologic mapping and isolation of autonomic ganglionic plexi. Ann Thorac Surg. 2007;83:538-41.

7. McClelland JH, Duke D, Reddy R. Preliminary results of a limited thoracotomy: new approach to treat atrial fibrillation. J Cardiovasc Electrophysiol. 2007;18:1289-95.

8. Edgerton JR, Jackman WM, Mack MJ. Minimally invasive pulmonary vein isolation and partial autonomic denervation for surgical treatment of atrial fibrillation. J Interv Card Electrophysiol. 2007;20:89-93.

9. Matsutani N, Takase B, Ozeki Y, Maehara T, Lee R. Minimally invasive cardiothoracic surgery for atrial fibrillation: a combined Japan-US experience. Circ J. 2008;72:434-6.

10. Edgerton JR, Edgerton ZJ, Weaver T, Reed K, Prince S, Herbert MA, et al. Minimally invasive pulmonary vein isolation and partial autonomic denervation for surgical treatment of atrial fibrillation. Ann Thorac Surg. 2008;86:35-8; discussion 39 .

11. Lonnerholm S, Blomstrom P, Nilsson L, Oxelbark S, Jideus L, BlomstromLundqvist C. Effects of the maze operation on health-related quality of life in patients with atrial fibrillation. Circulation. 2000;101:2607-11.

12. Pappone C, Santinelli V, Manguso F, Vicedomini G, Gugliotta F, Augello G, et al Pulmonary vein denervation enhances long-term benefit after circumferential ablation for paroxysmal atrial fibrillation. Circulation. 2004;109:327-34.

13. Scherlag BJ, Nakagawa H, Jackman WM, Yamanashi WS, Patterson E, Po S, et al. Electrical stimulation to identify neural elements on the heart: their role in atria fibrillation. J Interv Card Electrophysiol. 2005;13(Suppl 1):37-42.

14. Katritsis D, Giazitzoglou E, Sougiannis D, Goumas N, Paxinos G, Camm AJ. Anatomic approach for ganglionic plexi ablation in patients with paroxysmal atrial fibrillation. Am J Cardiol. 2008;102:330-4.

15. Oh S, Zhang Y, Bibevski S, Marrouche NF, Natale A, Mazgalev TN. Vagal denervation and atrial fibrillation inducibility: epicardial fat pad ablation does not have long-term effects. Heart Rhythm. 2006;3:701-8.

16. Bertaglia E, Zoppo F, Tondo C, Colella A, Mantovan R, Senatore G, et al. Early complications of pulmonary vein catheter ablation for atrial fibrillation: a multicenter prospective registry on procedural safety. Heart Rhythm. 2007;4:1265-71.

17. Pappone C, Oral H, Santinelli V, Vicedomini G, Lang CC, Manguso F, et al Atrio-esophageal fistula as a complication of percutaneous transcatheter ablation of atrial fibrillation. Circulation. 2004;109:2724-6.

18. Wudel JH, Chaudhuri P, Hiller JJ. Video-assisted epicardial ablation and left atrial appendage exclusion for atrial fibrillation: extended follow-up. Ann Thorac Surg. 2008;85:34-8. 